\title{
Identifying and Tracking Defects in Dynamic Supramolecular Polymers
}

Piero Gasparotto, ${ }^{*},$, Il Davide Bochicchio, ${ }^{\ddagger}$ Michele Ceriotti, ${ }^{*} \dagger$ and Giovanni M.

$$
\operatorname{Pavan}^{*} \$, \S
$$

$\dagger$ Laboratory of Computational Science and Modeling, Institute des Materiaux, Ecole polytechnique fédérale de Lausanne, CH-1015 Lausanne, Switzerland

$\ddagger$ Department of Innovative Technologies, University of Applied Sciences and Arts of Southern

Switzerland, Galleria 2, Via Cantonale 2c, CH-6928 Manno, Switzerland

IThomas Young Centre and Department of Physics and Astronomy, University College London, Gower Street London WC1E 6BT, United Kingdom

$\S$ Department of Applied Science and Technology, Politecnico di Torino, Corso Duca degli Abruzzi 24, 10129 Torino, Italy

E-mail: p.gasparotto@ucl.ac.uk; michele.ceriotti@epfl.ch; giovanni.pavan@polito.it 


\section{Supporting Information Available}

\section{Probabilistic Analysis of Molecular Motifs}

PAMM is a pattern recognition framework designed to identify molecular motifs based on their meta-stability. The core idea behind the PAMM scheme consists into partitioning the probability distribution function (PDF) of structures sampled from an atomistic simulation or collected from experiments. The input is simply a series of $N$ (preferably high-dimensional) vectors representing local or global environments. This could be any function of the atomic coordinates, such as distances, angles or more sophisticated descriptors. Then one needs to perform a kernel density estimation (KDE) on a grid extracted using farthest point sampling (FPS), which has proven to be well suited for selecting the most widely spread set of landmarks from the initial set. Combing FPS with KDE allows for linear scaling with the number of samples and for the use of very large datasets, which is usually a bottleneck for most clustering algorithms.

The KDE on a grid point $\mathbf{y}_{i}$, given a set of $\{\mathbf{x}\}$ initial observations, can be written as

$$
P\left(\mathbf{y}_{i}\right)=\frac{1}{\sum_{j=1}^{N} w_{j}} \sum_{j=1}^{N} w_{j} K_{\mathbf{H}_{j}}\left(\mathbf{x}_{j}-\mathbf{y}_{i}\right),
$$

where $w_{i}$ is simply a weight, $K_{\mathbf{H}_{j}}$ an anisotropic multivariate Gaussian kernel, and $\mathbf{H}_{j}$ is an adaptive bandwidth matrix. Two schemes have been suggested to optimally select the local kernel bandwidth. We here adopted the fixed localization window approach, scaling by a factor of 0.7 the automatically determined bandwidth. Having a reliable estimation of the probability density at each grid point, one can perform a density-based clustering to single out the different local maxima in the PDF and fit a multivariate Gaussian to each of the clusters obtained. In this way a GMM (Gaussian Mixture Modelling) can be used to represent the original PDF and define the so-called probabilistic motifs identifiers (PMIs), function of the coordinates giving a score between zero and one that represents the degree of confidence by which a new local structure can be assigned to each of the clusters. 
Given a feature vector $\mathbf{x}$, the PMI corresponding to the $k$ th cluster can be written as:

$$
\hat{P}_{k}(\mathbf{x})=p_{k} G(\mathbf{x}) /(\hat{P}(\mathbf{x}))
$$

where $\hat{P}(\mathbf{x})=\sum_{k=1}^{n} p_{k} G(\mathbf{x})$ is the GMM modelling the PDF, $G(\mathbf{x})$ is the multivariate Gaussian fitted to the $k$ th cluster and $p_{k}$ is its weight in the mixture. Finally, one can post-process any trajectory and use the predominant PMI to classify each monomer according to its structural state.

\section{Smooth Overlap of Atomic Positions}

SOAP is a general state-of-art atom-centered, density-based representation of the atomic environment which has been proven very powerful for both properties prediction and structural classification. A sum of Gaussians centered on each surrounding atom of species $\alpha$ produces a smooth representation of the atomic density around an atom $j$.

By defining a cutoff function $f_{c}$ defining the extent of the local environments, the surrounding atomic density can be mapped into a local probability amplitude $\psi_{\mathscr{X}_{j}}^{\alpha}(\mathbf{r})$ :

$$
\left\langle\alpha \mathbf{r} \mid \mathscr{X}_{j}\right\rangle \equiv \psi_{\mathscr{X}_{j}}^{\alpha}(\mathbf{r})=\sum_{i \in \alpha} f_{c}\left(\mathbf{r}_{i j}\right) g\left(\mathbf{r}-\mathbf{r}_{i j}\right)
$$

One can expand the local density in a basis of orthogonal radial basis functions $R_{n}(r)$ and spherical harmonics $Y_{m}^{l}(\hat{\mathbf{r}})$,

$$
\left\langle\alpha n l m \mid \mathscr{X}_{j}\right\rangle=\int \mathrm{d} \mathbf{r} R_{n}(r) Y_{m}^{l}(\hat{\mathbf{r}})\left\langle\alpha \mathbf{r} \mid \mathscr{X}_{j}\right\rangle
$$

This amplitude is invariant both to translations and permutations of atoms $\alpha$, but it is not rotationally invariant. By integrating over all relative rotations $\hat{R}$ tensor products between this atom density evaluated at different points, one can obtain a representation of the atomic environment that is spherical invariant, corresponding to $n$-body correlations between the atoms. In particular, the second-order (three-body) invariant can be easily computed, on a radial basis, in terms of the 
spherical decomposition 4, and corresponds to the SOAP power spectrum

$$
\left\langle\mathscr{X}_{j} \mid \alpha n \alpha^{\prime} n^{\prime} l\right\rangle \propto \frac{1}{\sqrt{2 l+1}} \sum_{m}(-1)^{m}\left\langle\alpha n l m \mid \mathscr{X}_{j}\right\rangle\left\langle\alpha^{\prime} n^{\prime} l-m \mid \mathscr{X}_{j}\right\rangle .
$$

We use $\left\langle\alpha n \alpha^{\prime} n^{\prime} l \mid \mathscr{X}_{k}\right\rangle$ as an agnostic descriptor to fully characterize the local environment surrounding each monomer in the fibers.

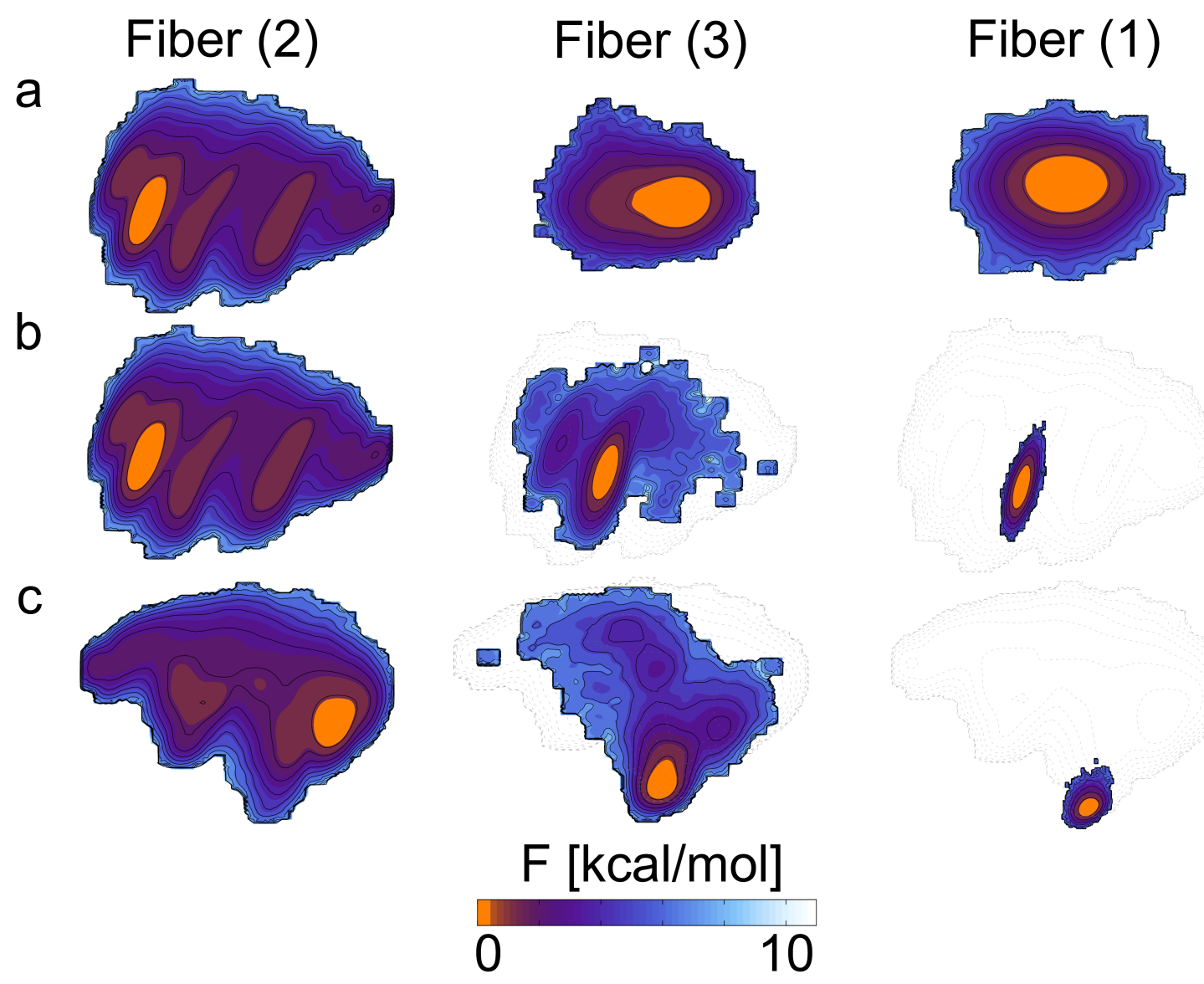

Figure S1: Low-dimensional FES for the different fibers using a PCA model (a) trained separately for the three fibers, (b) trained considering only fiber (2) (i.e. the system with the higher structural variability), and (c) trained considering the full dataset (no labels). Gray dashed lines refer to the contours of the corresponding Fiber (2)'s projection. 


\section{Low-dimensional embedding}

PCA is useful to extract important features from a high-dimensional dataset and reduce its dimensionality. The algorithm seeks a linear combination of variables such that the maximum variance is extracted from the input features. It then removes this variance and seeks a second linear combination which explains the maximum proportion of the remaining variance, and so on. One can then use the first $d$ principal components to embed a $D$-dimensional manifold into a $d$-dimensional one (with $d<<D$ ). To be fully agnostic and as general as possible, we chose to discard any prior knowledge about different fibers and train our PCA on the full dataset containing the SOAP vectors computed from all the three systems. However, it is interesting to check how the PCA trained on a single fiber performs in embedding the high-dimensional features from other fibers. Figure S1, shows the low-dimensional projections for the three fibers training the PCA on the total dataset (Figure S1c), only on fiber (2) (Figure S1b) (which is the fiber presenting the largest structural variability) and on the single fibers separately. One can see how training the model in the system with the higher degree of disorder can be enough to learn how to embed different (possibly more ordered) systems not seen during the training stage.

\section{PCA convergence}

One can estimate the minimum number of principal components to be used to sparsify SOAP vectors by considering the amount of variance in the original variables accounted for by each component. Figure S2 shows the percentage of cumulative proportion of variance explained by an increasing amount of principal components. We chose to use 8 components since it is enough to recover more than $90 \%$ of the initial variance for the full data set (without distinguishing different fibers).

\section{Choice of structural parameters}

SOAP descriptors allow us to adopt a general structural description for local atomic environments avoiding any possible prior human bias (angles, distances, gyration radius or other educated guesses). 


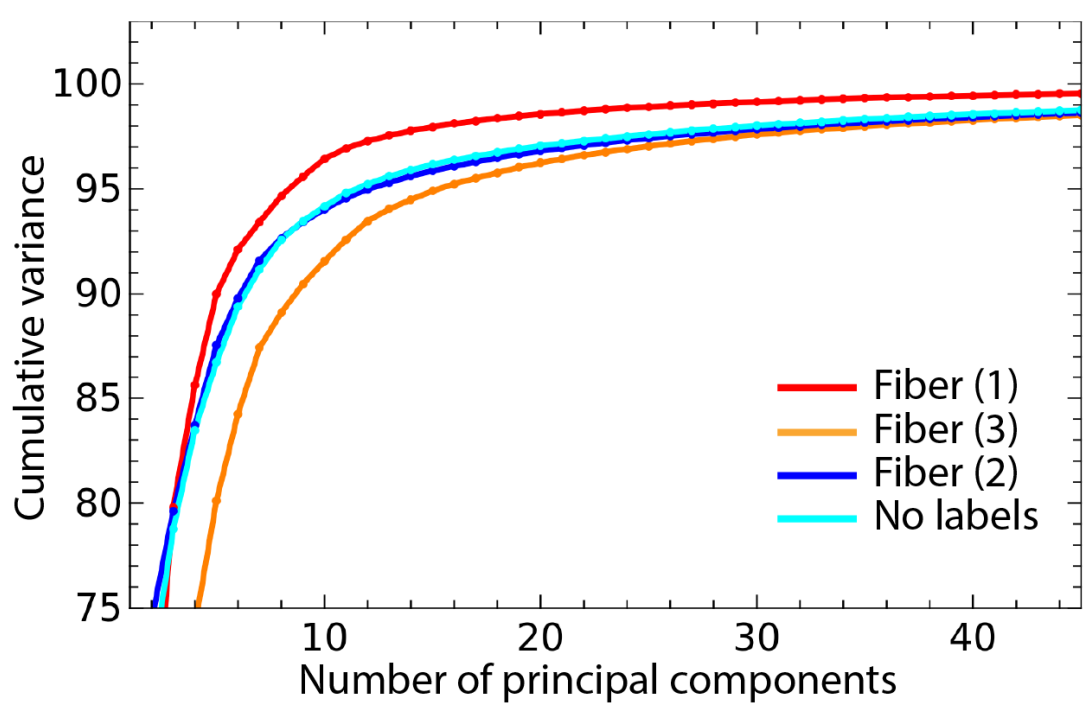

Figure S2: Cumulative variance as function of the number of principal components for the different fibers as well for the full dataset obtained removing the labels of the fibers.

However, few parameters must still be set in order to define the range of structural correlations captured by the SOAP vectors. In the specific, one has to guess a reasonable cut-off distance which has to be large enough to capture all the important physics, but also reasonably small in order to reduce at maximum the computational resources needed to compute and store the SOAP vectors. A good way to rapidly estimate the extent of local structural correlations is to use the Radial Distribution Function (RDF). To compute the RDF we considered only the center of mass of the aromatic ring of each monomer unit. Figure S3 shows that the first neighbour is typically at a distance lower than $6.0 \AA$.

We used the following parameters: cutoff $=8.0 \AA$, cutoff_tr_width $=0.5$, atom_sigma $=$ $1, \mathrm{n} \_\max =8,1 \_\max =8$. All other parameters were set to the default values. Explanations of the parameters are given in the quippy library reference, available at https://libatoms.github. io/QUIP/descriptors.html.

We tested different cutoff radius in order to find the minimum distance capable of capturing the structural complexity of the fibers, as well as to distinguish between different systems. As before, in this analysis we considered only the center of mass of the aromatic rings for computing the SOAP vectors using different cut-off distances. We then computed the PCA on the full datasets 


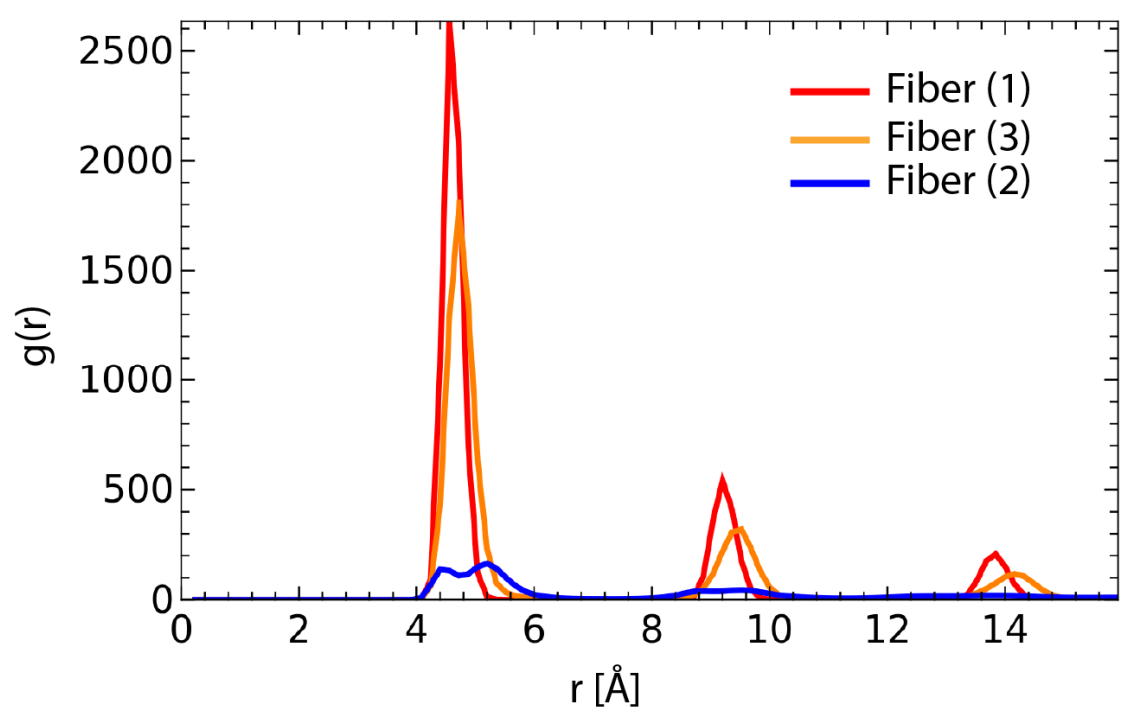

Figure S3: Comparing RDFs for fibers (2), (3) and (1). The RDF is computed considering only the center of mass of each aromatic ring in the monomer.

(removing the labels distinguishing among different fibers) and projected the SOAP features for the three systems onto different maps using the first two principal components. We also checked the robustness of our analysis by comparing the results obtained using cutoffs of $8 \AA, 12 \AA$ or $16 \AA$. In Figure S4, one can see that using cutoff $=8.0$ is enough to capture the different degree of complexity and population of the states in the three fibers.

\section{Automatic learning of defects}

This paper introduces a general method for automatic identification and classification of defects across different classes of supramolecular polymers. In our protocol, in order to make the analysis comparable between different fibers while keeping a detailed view on the stacking of the monomers, we considered the full aromatic ring plus the amide beads of each monomer. Here we show, however, that one could simplify even more the approach and consider only a virtual atom centered at the center of each monomer. Figure S5 shows that using only the information about the centers to build the SOAP vectors could be enough to capture qualitatively all the structural complexity between different fibers. To compute the SOAPs we used the parameters introduced before with cutoff $=8.0 \AA$, while the two-dimensional maps were produced projecting the SOAPs onto the first two 

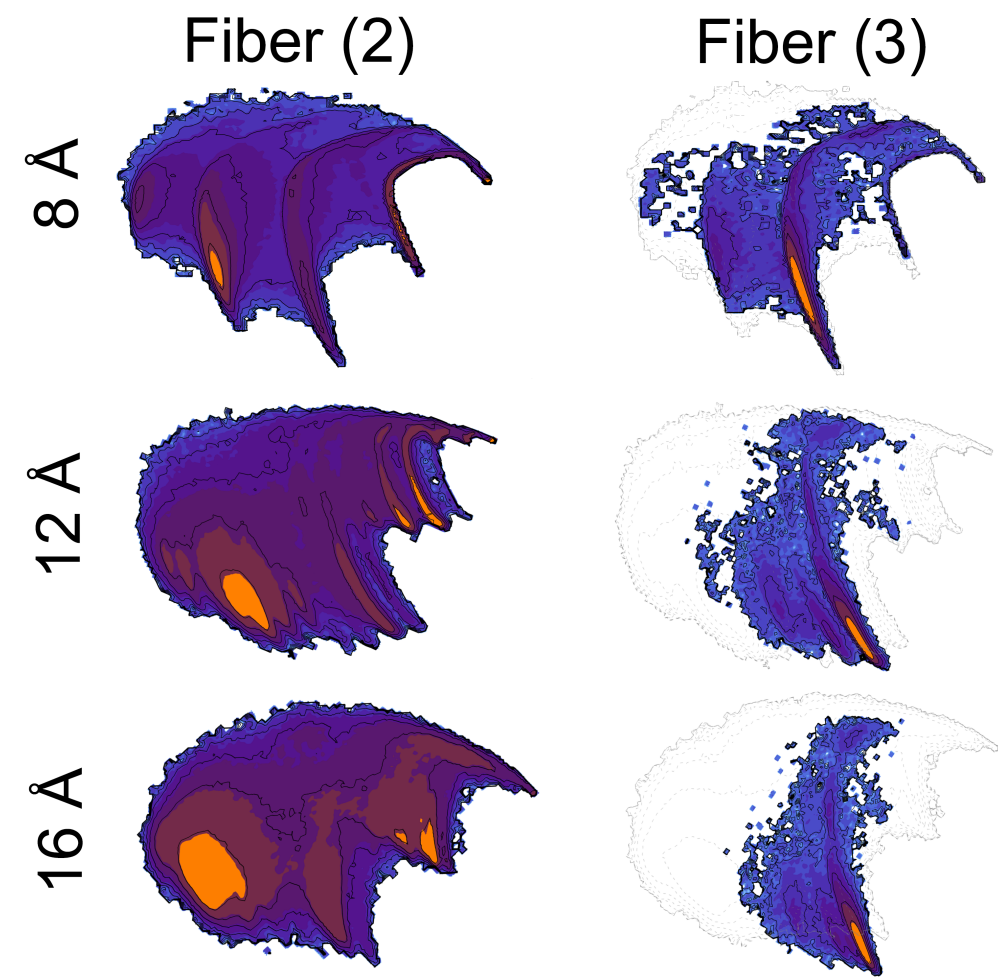

Fiber (1)
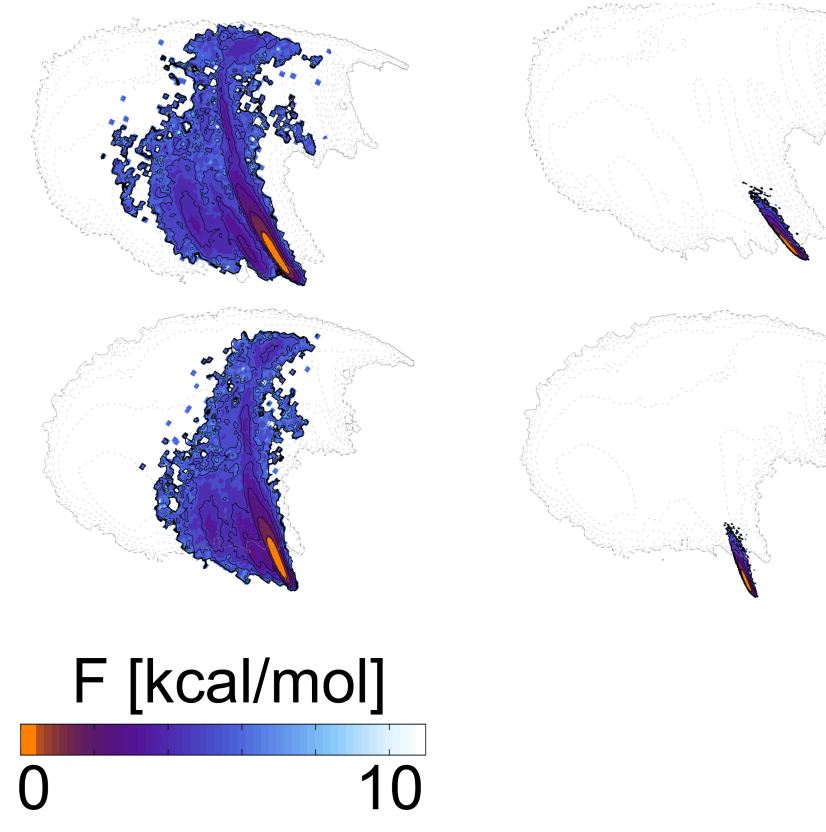

Figure S4: PCA projection of the SOAP vectors for the different systems using cutoff $=8.0$, cutoff $=12.0$, cutoff $=16.0$. The SOAP vectors are computed considering only the center of mass of each aromatic ring in the monomer. In gray are the contours of Fiber (2), which is structurally richer than the other fibers and help the reader to compare the different systems. 
principal components obtained from the PCA of the full dataset (without labels for different fibers). This analysis shows the power of SOAP when combined with PAMM for building a completely agnostic, data-driven approach to learn the structural complexity of polymers. Without having any prior knowledge on the systems one could start simply replacing each monomer with a point and study the correlations among those to capture most of the structural and the dynamical nature of the fiber.

Fiber (2)
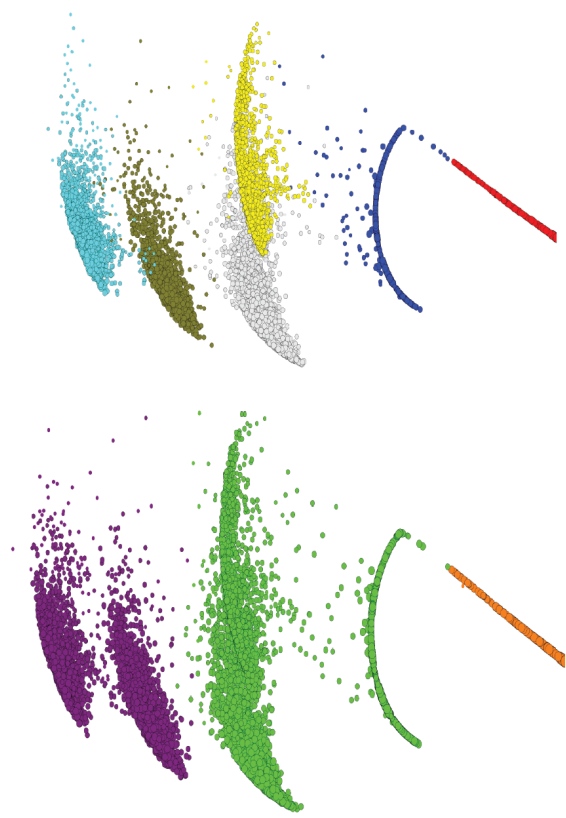

Fiber (3)
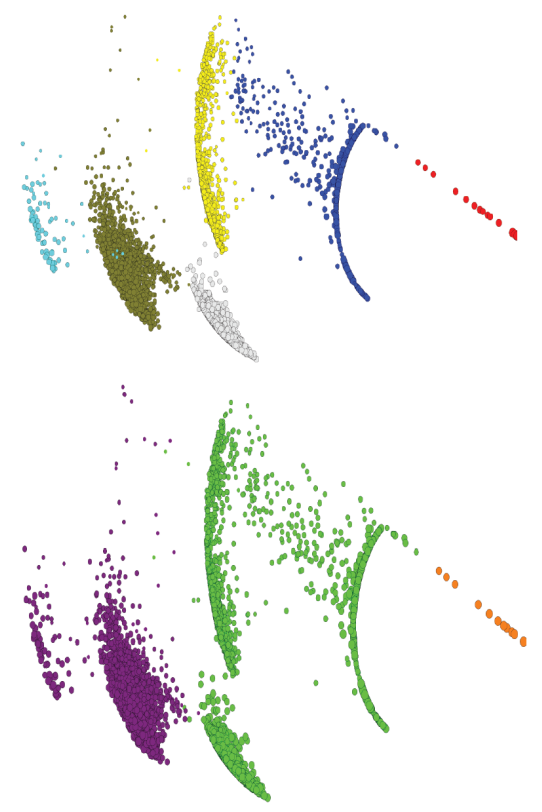

Fiber (1)
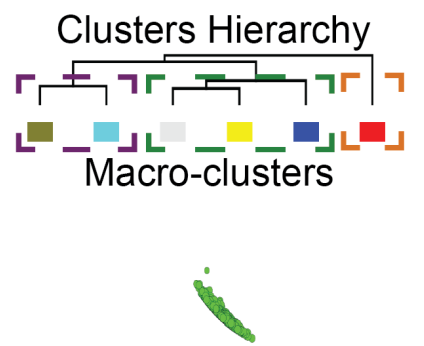

Figure S5: Projection of PAMM clustering of SOAP vectors computed using only the centers of the aromatic rings. 